\title{
Prasugrel versus Clopidogrel for Acute Coronary Syndromes without Revascularization
}

\author{
Matthew T. Roe, M.D., M.H.S., Paul W. Armstrong, M.D., Keith A.A. Fox, M.B., Ch.B., \\ Harvey D. White, M.B., Ch.B., D.Sc., Dorairaj Prabhakaran, M.D., D.M., \\ Shaun G. Goodman, M.D., Jan H. Cornel, M.D., Ph.D., Deepak L. Bhatt, M.D., M.P.H., \\ Peter Clemmensen, M.D., D.M.Sc., Felipe Martinez, M.D., Diego Ardissino, M.D., \\ Jose C. Nicolau, M.D., Ph.D., William E. Boden, M.D., Paul A. Gurbel, M.D., \\ Witold Ruzyllo, M.D., Anthony J. Dalby, M.D., Darren K. McGuire, M.D., M.H.Sc., \\ Jose L. Leiva-Pons, M.D., Alexander Parkhomenko, M.D., Ph.D., Shmuel Gottlieb, M.D., \\ Gracita O. Topacio, M.D., Christian Hamm, M.D., Gregory Pavlides, M.D., \\ Assen R. Goudev, M.D., Ali Oto, M.D., Chuen-Den Tseng, M.D., Ph.D., \\ Bela Merkely, M.D., Ph.D., D.Sc., Vladimir Gasparovic, M.D., Ph.D., Ramon Corbalan, M.D., \\ Mircea Cinteză, M.D., Ph.D., R. Craig McLendon, R.N., Kenneth J. Winters, M.D., \\ Eileen B. Brown, Ph.D., Yuliya Lokhnygina, Ph.D., Philip E. Aylward, B.M., B.Ch., Ph.D., \\ Kurt Huber, M.D., Judith S. Hochman, M.D., and E. Magnus Ohman, M.B., Ch.B., \\ for the TRILOGY ACS Investigators:
}

\section{A B STRACT}

\section{BACKGROUND}

The effect of intensified platelet inhibition for patients with unstable angina or myocardial infarction without ST-segment elevation who do not undergo revascularization has not been delineated.

\section{METHODS}

In this double-blind, randomized trial, in a primary analysis involving 7243 patients under the age of 75 years receiving aspirin, we evaluated up to 30 months of treatment with prasugrel (10 mg daily) versus clopidogrel ( $75 \mathrm{mg}$ daily). In a secondary analysis involving 2083 patients 75 years of age or older, we evaluated $5 \mathrm{mg}$ of prasugrel versus $75 \mathrm{mg}$ of clopidogrel.

\section{RESULTS}

At a median follow-up of 17 months, the primary end point of death from cardiovascular causes, myocardial infarction, or stroke among patients under the age of 75 years occurred in $13.9 \%$ of the prasugrel group and $16.0 \%$ of the clopidogrel group (hazard ratio in the prasugrel group, $0.91 ; 95 \%$ confidence interval [CI], 0.79 to $1.05 ; \mathrm{P}=0.21$ ). Similar results were observed in the overall population. The prespecified analysis of multiple recurrent ischemic events (all components of the primary end point) suggested a lower risk for prasugrel among patients under the age of 75 years (hazard ratio, $0.85 ; 95 \% \mathrm{CI}, 0.72$ to $1.00 ; \mathrm{P}=0.04$ ). Rates of severe and intracranial bleeding were similar in the two groups in all age groups. There was no significant between-group difference in the frequency of nonhemorrhagic serious adverse events, except for a higher frequency of heart failure in the clopidogrel group.

\section{CONCLUSIONS}

Among patients with unstable angina or myocardial infarction without ST-segment elevation, prasugrel did not significantly reduce the frequency of the primary end point, as compared with clopidogrel, and similar risks of bleeding were observed. (Funded by Eli Lilly and Daiichi Sankyo; TRILOGY ACS ClinicalTrials.gov number, NCT00699998.)
The authors' affiliations are listed in the Appendix. Address reprint requests to Dr. Roe at Duke Clinical Research Institute, 2400 Pratt St., Rm. 7035, Durham, NC 27705, or at matthew.roe@duke.edu.

*The Targeted Platelet Inhibition to Clarify the Optimal Strategy to Medically Manage Acute Coronary Syndromes (TRILOGY ACS) investigators are listed in the Supplementary Appendix, available at NEJM.org.

This article was published on August 26, 2012, and updated on August 27, 2012, at NEJM.org.

N EnglJ Med 2012;367:1297-309. DOI: 10.1056/NEJMoal205512

Copyright (C) 2012 Massachusetts Medical Society. 
LINICAL-PRACTICE GUIDELINES FOR PAtients with acute coronary syndromes consisting of unstable angina or myocardial infarction without ST-segment elevation recommend a strategy of early invasive management (angiography within 48 to 72 hours with provisional revascularization) for patients at moderate to high risk. ${ }^{1,2}$ However, analyses from clinical trials and national registries have shown that many such patients are treated medically without revascularization and that such patients have poorer long-term cardiovascular outcomes than those who undergo revascularization. ${ }^{3-6}$ Even though patients with acute coronary syndromes who receive only medical therapy have an increased-risk profile, they have been underrepresented in large-scale, contemporary, randomized trials..$^{7-9}$

Given the previously demonstrated benefits of prasugrel versus clopidogrel (both thienopyridine inhibitors of the platelet $\mathrm{P} 2 \mathrm{Y} 12$ receptor) among patients undergoing percutaneous coronary intervention (PCI), we evaluated whether aspirin plus prasugrel is superior to aspirin plus clopidogrel for long-term therapy in patients with unstable angina or myocardial infarction without ST-segment elevation who were under the age of 75 years. ${ }^{9}$ We also undertook a concomitant and exploratory assessment of a lower prasugrel dose for patients 75 years of age or older.

\section{METHODS}

\section{STUDY DESIGN}

The Targeted Platelet Inhibition to Clarify the Optimal Strategy to Medically Manage Acute Coronary Syndromes (TRILOGY ACS) study was a randomized, double-blind, double-dummy, activecontrol, event-driven trial. The study design has been described previously, ${ }^{10}$ and the protocol is available with the full text of this article at NEJM .org. The executive and steering committees, which included academic investigators and representatives of the sponsor (Eli Lilly and Daiichi Sankyo), designed the study and supervised its conduct. (A complete list of committee members is provided in the Supplementary Appendix, available at NEJM .org.) An independent data and safety monitoring board evaluated the safety of patients with semiannual meetings during the trial. Study data were collected and managed by Quintiles. Statistical analyses were performed independently by the academic coordinating center at the Duke
Clinical Research Institute. The first draft of the manuscript was written jointly by the study's principal investigator and chair. The academic representatives of the executive and steering committees contributed to subsequent manuscript drafts and approved the submission of the final manuscript for publication. The study's principal investigator and chair had full access to all data, verified their accuracy, and vouch for the fidelity of the study to the protocol. The study was approved by the national regulatory authority in each participating country and by the local ethics committee or institutional review board at each study center.

\section{STUDY PATIENTS}

Patients with acute coronary syndromes were eligible if they were selected for a final treatment strategy of medical management without revascularization within 10 days after the index event. Patients with myocardial infarction without STsegment elevation had elevated cardiac markers, whereas patients with unstable angina with negative cardiac markers had an ST-segment depression of more than $1 \mathrm{~mm}$ in two or more electrocardiographic leads. Patients were required to have at least one of four risk criteria: an age of at least 60 years, the presence of diabetes mellitus, previous myocardial infarction, or previous revascularization with either PCI or coronary-artery bypass grafting (CABG). Angiography was not required for enrollment, but if such a procedure was planned, it had to be performed before randomization. Patients who underwent angiography were required to have evidence of coronary disease (native coronary stenosis of $>30 \%$ or previous PCI or CABG). Major exclusion criteria included a history of transient ischemic attack or stroke, PCI or CABG within the previous 30 days, renal failure requiring dialysis, and concomitant treatment with an oral anticoagulant.

From June 27, 2008, through September 12, 2011, we enrolled 9326 patients at 966 sites in 52 countries. Across regions, 3090 participants were enrolled in Central and Eastern Europe (33.1\% of the total), 994 in Western Europe and Scandinavia (10.7\%), 1276 in Latin America (13.7\%), 752 in East Asia (8.1\%), 1141 in India (12.2\%), 1271 in North America (13.6\%), 658 in the Mediterranean area (7.1\%), and 144 in Australia, New Zealand, and South Africa (1.5\%). A total of 7243 patients were younger than 75 years of age (77.7\%), whereas 2083 patients were 75 years of age or older 
(22.3\%). All patients provided written informed consent.

\section{STUDY TREATMENT}

Patients were randomly assigned to receive either prasugrel or clopidogrel in a double-blind, doubledummy fashion with the use of an interactive voiceresponse system. Patients who underwent randomization within 72 hours after the first medical contact without previous clopidogrel treatment received a loading dose of $30 \mathrm{mg}$ of prasugrel or $300 \mathrm{mg}$ of clopidogrel, which was followed by daily blinded maintenance administration of a study drug. Patients who did not undergo randomization within 72 hours were required to be treated with open-label clopidogrel before randomization and were started on daily maintenance administration of a study drug after randomization.

The prasugrel maintenance dose was $10 \mathrm{mg}$, which was adjusted to $5 \mathrm{mg}$ for patients who were 75 years of age or older or who weighed less than $60 \mathrm{~kg}$. The clopidogrel maintenance dose was $75 \mathrm{mg}$ for all patients. Pharmacokinetic modeling from previous trials showed that $5 \mathrm{mg}$ of prasugrel in patients weighing less than $60 \mathrm{~kg}$ resulted in an antiplatelet effect that was similar to that for $10 \mathrm{mg}$ in heavier patients. ${ }^{11}$ The 5 -mg dose that was used in participants 75 years of age or older had not been evaluated in previous outcomes trials. Concomitant treatment with aspirin was required, and a daily dose of $100 \mathrm{mg}$ or less was strongly recommended. Study treatments continued for a minimum of 6 months and a maximum of 30 months.

\section{END POINTS}

The primary efficacy end point was a composite of death from cardiovascular causes, nonfatal myocardial infarction, or nonfatal stroke among patients under the age of 75 years. Other end points have been defined previously. ${ }^{10}$ Suspected ischemic and bleeding end points were evaluated by an independent cardiovascular adjudication committee whose members were unaware of studygroup assignments. Suspected new, nonbenign neoplasm end points were adjudicated by an independent oncology adjudication committee (for details, see the Supplementary Appendix).

\section{BLEEDING EVENTS}

Key bleeding end points were analyzed on the basis of Global Use of Strategies to Open Occluded
Coronary Arteries (GUSTO) criteria for severe or life-threatening bleeding not related to CABG and Thrombolysis in Myocardial Infarction (TIMI) criteria for major bleeding not related to CABG.

\section{STATISTICAL ANALYSIS}

We estimated that 688 patients with primary efficacy events would be needed to ensure a power of $90 \%$ to detect a relative risk reduction of $22 \%$ between the two study groups among patients under the age of 75 years using a two-sided test at the $5 \%$ significance level. Formal sample-size analyses were not performed for patients who were 75 years of age or older, since this secondary analysis was exploratory, with a previously untested dose of prasugrel (5 mg daily); however, an enrollment of more than 2000 patients was targeted. All efficacy analyses were performed on the intention-to-treat population.

Testing for the superiority of prasugrel over clopidogrel was done with a two-sided log-rank test and stratified according to clopidogrel status at the time of randomization, as described previously. ${ }^{10}$ If superiority was established in patients under the age of 75 years, then testing for superiority would have been done in a hierarchical manner on the overall patient cohort, stratified according to age group. We explored the consistency of treatment effect on the primary efficacy end point in prespecified subgroups.

Landmark analyses of the primary end point were not prespecified. An Andersen-Gill intensitymodel analysis using a robust variance estimate was prespecified and performed to account for repeated ischemic events among all components of the primary end point for the overall period and using a time-dependent model with separate hazard ratios before and after 30 days, 6 months, and 12 months. ${ }^{12}$

Key bleeding end points were evaluated in patients who received at least one dose of a study drug, with a stratified log-rank test during the period from the initiation of the study drug until 7 days after its discontinuation. New, nonbenign neoplasm end points were evaluated in the overall population in patients who received at least one dose of a study drug.

During systematic audits of study centers, four sites (enrolling 120 patients) were found to have violated key protocol requirements and Good Clinical Practice guidelines. These sites were closed, and the administration of study drugs to 
all patients at those sites was discontinued. The executive committee decided to prospectively exclude these patients from all analyses before unblinding and database lock.

\section{RESULTS}

\section{STUDY PATIENTS}

Of 9326 patients who underwent randomization, 573 did not complete the study; vital status was collected on all but 18 patients (Fig. S1 in the Supplementary Appendix). The median duration of exposure to a study drug was 14.8 months (interquartile range, 8.2 to 23.6). During the follow-up period, $76 \%$ of patients in the prasugrel group con- tinued to receive the study drug, as compared with $78 \%$ of those in the clopidogrel group $(\mathrm{P}=0.03)$. The median duration of follow-up for all patients in the trial was 17.1 months (interquartile range, 10.4 to 24.4$)$.

Baseline characteristics were generally balanced in the two study groups among patients under the age of 75 years and in the overall population (Table 1). Among patients under the age of 75 years, the median time from presentation to the initiation of a study drug was just over 4 days, and nearly half the patients underwent angiography before randomization.

During follow-up, 571 of the 7243 patients under the age of 75 years $(7.9 \%)$ underwent revasculari-

\begin{tabular}{|c|c|c|c|c|}
\hline \multirow[t]{2}{*}{ Characteristic } & \multicolumn{2}{|c|}{ Age $<75$ Years } & \multicolumn{2}{|c|}{ Overall Population } \\
\hline & $\begin{array}{l}\text { Prasugrel } \\
(\mathrm{N}=3620)\end{array}$ & $\begin{array}{c}\text { Clopidogrel } \\
(\mathrm{N}=3623)\end{array}$ & $\begin{array}{l}\text { Prasugrel } \\
(\mathrm{N}=4663)\end{array}$ & $\begin{array}{r}\text { Clopidogre } \\
(\mathrm{N}=4663)\end{array}$ \\
\hline \multicolumn{5}{|l|}{ Age (yr) } \\
\hline Median & 62 & 62 & 66 & 66 \\
\hline Interquartile range & $56-68$ & $56-68$ & $58-74$ & $59-73$ \\
\hline Female sex (\%) & 36.2 & 35.6 & 39.2 & 39.1 \\
\hline Body weight <60 kg (\%) & 13.1 & 12.8 & 15.2 & 14.9 \\
\hline \multicolumn{5}{|l|}{ Disease classification (\%) } \\
\hline NSTEMI & 67.8 & 67.2 & 70.4 & 69.4 \\
\hline Unstable angina & 32.2 & 32.8 & 29.6 & 30.6 \\
\hline Killip class II to IV on presentation (\%) & 9.5 & 10.3 & 12.1 & 12.2 \\
\hline \multicolumn{5}{|c|}{ Time from presentation until start of study drug (hr) } \\
\hline Median & 102 & 103 & 108 & 108 \\
\hline Interquartile range & $58-158$ & $60-157$ & $62-160$ & $63-160$ \\
\hline \multicolumn{5}{|l|}{ Cardiovascular risk factors (\%) } \\
\hline Family history of coronary artery disease & 31.5 & 32.1 & 29.7 & 31.1 \\
\hline Hypertension & 80.3 & 80.4 & 81.9 & 82.0 \\
\hline Hyperlipidemia & 58.2 & 59.7 & 59.0 & 59.3 \\
\hline Diabetes mellitus & 38.5 & 39.3 & 37.7 & 38.3 \\
\hline Current or recent smoker & 23.3 & 23.6 & 19.7 & 20.2 \\
\hline \multicolumn{5}{|l|}{ Cardiovascular disease history (\%) } \\
\hline Previous myocardial infarction & 43.3 & 44.8 & 42.9 & 43.3 \\
\hline Previous $\mathrm{PCl}$ & 27.0 & 29.1 & 25.6 & 26.7 \\
\hline Previous CABG & 14.6 & 16.3 & 15.2 & 16.1 \\
\hline Previous peripheral arterial disease & 6.0 & 7.3 & 7.2 & 7.6 \\
\hline Previous atrial fibrillation & 5.9 & 6.2 & 7.6 & 8.0 \\
\hline Previous heart failure & 17.1 & 17.1 & 17.6 & 17.6 \\
\hline \multicolumn{5}{|l|}{ GRACE risk scoretr } \\
\hline Median & 114 & 115 & 122 & 121 \\
\hline Interquartile range & $101-128$ & $102-128$ & $105-140$ & $106-138$ \\
\hline
\end{tabular}




\begin{tabular}{|c|c|c|c|c|}
\hline \multirow[t]{2}{*}{ Characteristic } & \multicolumn{2}{|c|}{ Age $<75$ Years } & \multicolumn{2}{|c|}{ Overall Population } \\
\hline & $\begin{array}{l}\text { Prasugrel } \\
(\mathrm{N}=3620)\end{array}$ & $\begin{array}{l}\text { Clopidogrel } \\
(\mathrm{N}=3623)\end{array}$ & $\begin{array}{l}\text { Prasugrel } \\
(\mathrm{N}=4663)\end{array}$ & $\begin{array}{c}\text { Clopidogrel } \\
(\mathrm{N}=4663)\end{array}$ \\
\hline \multicolumn{5}{|l|}{ Creatinine clearance ( $\mathrm{ml} / \mathrm{min})$} \\
\hline Median & 81 & 81 & 73 & 73 \\
\hline Interquartile range & $63-104$ & $63-102$ & $54-97$ & $54-96$ \\
\hline \multicolumn{5}{|l|}{ Prerandomization clopidogrel stratum (\%)』 } \\
\hline 1 & 4.2 & 4.6 & 4.2 & 4.4 \\
\hline 2 & 69.3 & 68.4 & 69.9 & 69.8 \\
\hline \multicolumn{5}{|c|}{$\begin{array}{l}\text { Time from start of clopidogrel until start of study } \\
\text { drug for participants in stratum } 2(\mathrm{hr})\end{array}$} \\
\hline Median & 103 & 105 & 107 & 108 \\
\hline Interquartile range & $64-154$ & $61-153$ & $65-155$ & $63-155$ \\
\hline 3 & 26.5 & 27.0 & 25.9 & 25.9 \\
\hline Angiography performed before randomization (\%) & 42.1 & 43.1 & 41.2 & 41.4 \\
\hline \multicolumn{5}{|l|}{ Concomitant medication at randomization (\%) } \\
\hline \multicolumn{5}{|l|}{ Aspirin } \\
\hline$<100 \mathrm{mg} /$ day & 34.6 & 33.5 & 33.9 & 32.8 \\
\hline $100-250 \mathrm{mg} /$ day & 52.2 & 52.3 & 53.1 & 53.2 \\
\hline$>250 \mathrm{mg}$ & 7.2 & 8.1 & 7.0 & 7.4 \\
\hline Beta-blocker & 78.1 & 77.5 & 78.3 & 77.2 \\
\hline ACE inhibitor or angiotensin-receptor blocker & 74.9 & 75.1 & 75.3 & 75.4 \\
\hline Statin & 83.9 & 84.0 & 83.6 & 83.1 \\
\hline Proton-pump inhibitor & 22.9 & 23.1 & 25.3 & 25.0 \\
\hline
\end{tabular}

* For patients under the age of 75 years, significant between-group differences $(P<0.05)$ were observed only for previous percutaneous coronary intervention $(\mathrm{PCl})$, previous coronary-artery bypass grafting (CABG), and previous peripheral arterial disease. For the overall population, there were no significant between-group differences. ACE denotes angiotensinconverting enzyme, and NSTEMI non-ST-segment elevation myocardial infarction.

$\dagger$ This category was defined as cigarette smoking within 30 days before randomization.

$\lceil$ Global Registry of Acute Coronary Events (GRACE) risk scores range from 0 to 372, with a score of 140 or more considered to indicate high risk.

$\int$ Clopidogrel strata are defined as follows: stratum 1, no clopidogrel administered before randomization, with randomization occurring within 72 hours after the first medical contact; stratum 2, a loading dose of clopidogrel (300 to $600 \mathrm{mg})$ administered for the index event, followed by a daily clopidogrel maintenance dose $(75 \mathrm{mg})$ until the day of randomization; and stratum 3, a daily maintenance dose of clopidogrel $(75 \mathrm{mg})$, starting at least 5 days before presentation for the index event and continuing until the day of randomization.

zation (427 underwent PCI, 170 underwent CABG, and 26 underwent both procedures), with a median time from randomization to first revascularization of 113 days (interquartile range, 40 to 334).

\section{EFFICACY}

At 30 months, there was no significant betweengroup difference in the rate of the primary end point among the primary cohort of patients under the age of 75 years (Table 2). At a median follow-up of 17 months, the primary end point occurred in $13.9 \%$ of the prasugrel group and $16.0 \%$ of the clopidogrel group (hazard ratio in the prasugrel group, 0.91 ; $95 \%$ confidence inter- val [CI], 0.79 to $1.05 ; \mathrm{P}=0.21$ ) (Fig. 1A). Because superiority was not established in this cohort, the prespecified testing strategy did not direct further superiority testing, but efficacy and safety results for the overall cohort (all ages) are presented for completeness (Tables 2 and 3, and Table S1 and Fig. S2 in the Supplementary Appendix). Among patients under the age of 75 years, the KaplanMeier curves for the primary end point overlapped until approximately 12 months, after which the curves diverged. Similar observations were made for each of the secondary end points (death from cardiovascular causes, all myocardial infarctions, and strokes) (Fig. 1B, 1C, and 1D). 


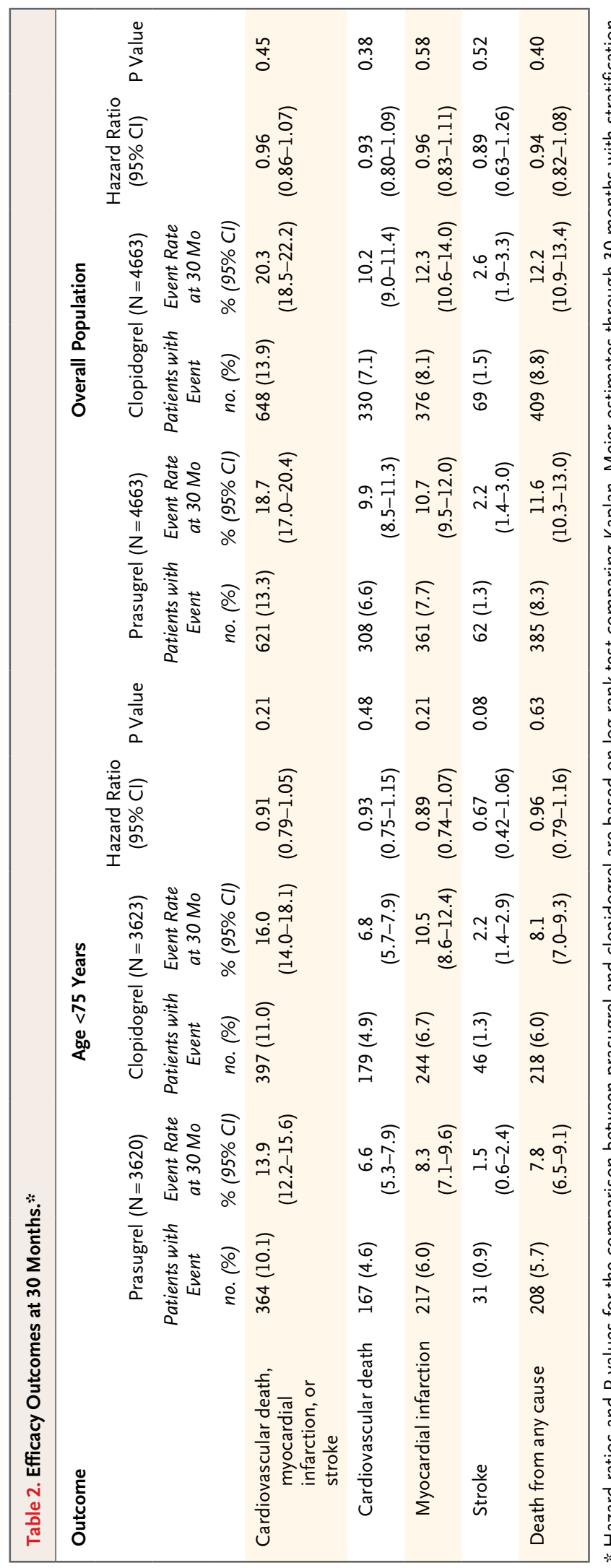

Because we observed a divergence of treatment effect among patients under the age of 75 years after the prespecified 12-month time point, we tested the difference in treatment effect between the first 12 months and subsequent months in a post hoc analysis using a time-dependent Cox proportional-hazards model; in this analysis, the time period and the interaction between the time period and treatment were time-dependent factors. The frequency of the primary end point through 12 months was similar among study groups, with a weak trend toward a reduced risk in the prasugrel group after 12 months $(\mathrm{P}=0.07$ for interaction) (Fig. 1A).

The frequency of the primary end point in the two study groups did not differ significantly among prespecified subgroups of patients who were under the age of 75 years, but an interaction with prasugrel treatment was apparent in current or recent smokers, those who underwent angiography before randomization, and those taking a proton-pump inhibitor at randomization (Fig. 2).

The prespecified analysis that was performed to account for multiple recurrent ischemic events suggested a lower risk among patients under the age of 75 years in the prasugrel group (hazard ratio, $0.85 ; 95 \% \mathrm{CI}, 0.72$ to $1.00 ; \mathrm{P}=0.04$ ). Among patients who had an ischemic event, 364 patients in the prasugrel group (10.1\%) had at least one ischemic event, as compared with 397 patients in the clopidogrel group (11.0\%), whereas 77 (2.1\%) versus $109(3.0 \%)$ had at least two recurrent ischemic events, and $18(0.5 \%)$ versus $24(0.7 \%)$ had at least three recurrent ischemic events, respectively. (Data on the breakdown of component end points per category of number of recurrent events are provided in Table S2 in the Supplementary Appendix.) In the time-dependent analysis of recurrent events using a 12-month landmark time point, there was a significant interaction with treatment and time $(\mathrm{P}=0.02)$. The risk of recurrent ischemic events in the prasugrel group was lower after 12 months (hazard ratio for $<12$ months, 0.94 [95\% CI, 0.79 to 1.12 ], vs. hazard ratio for $\geq 12$ months, 0.64 [95\% CI, 0.48 to 0.86$])$.

\section{SAFETY}

At 30 months, the key bleeding end points of nonCABG-related severe or life-threatening events (according to GUSTO criteria) and major bleeding 


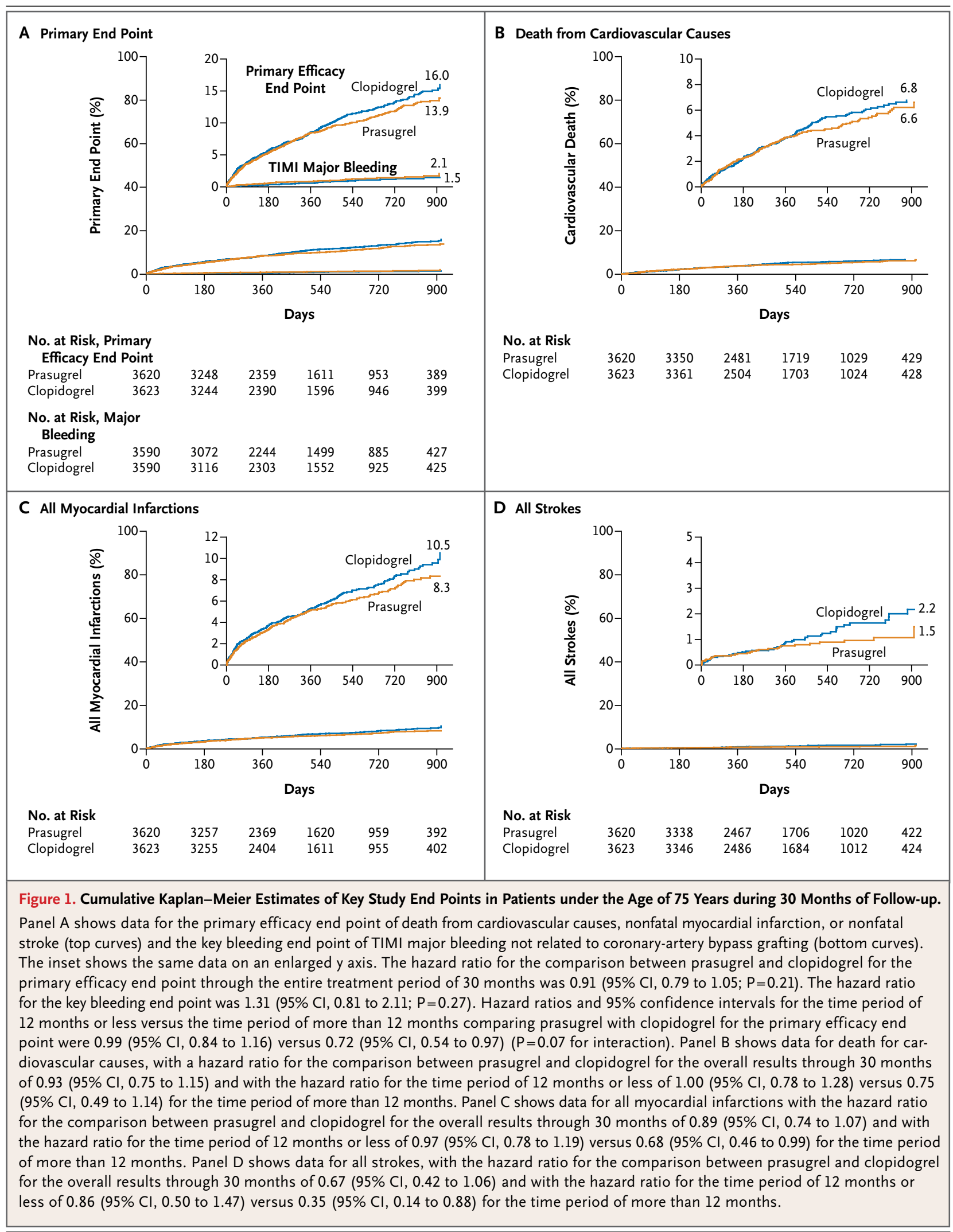




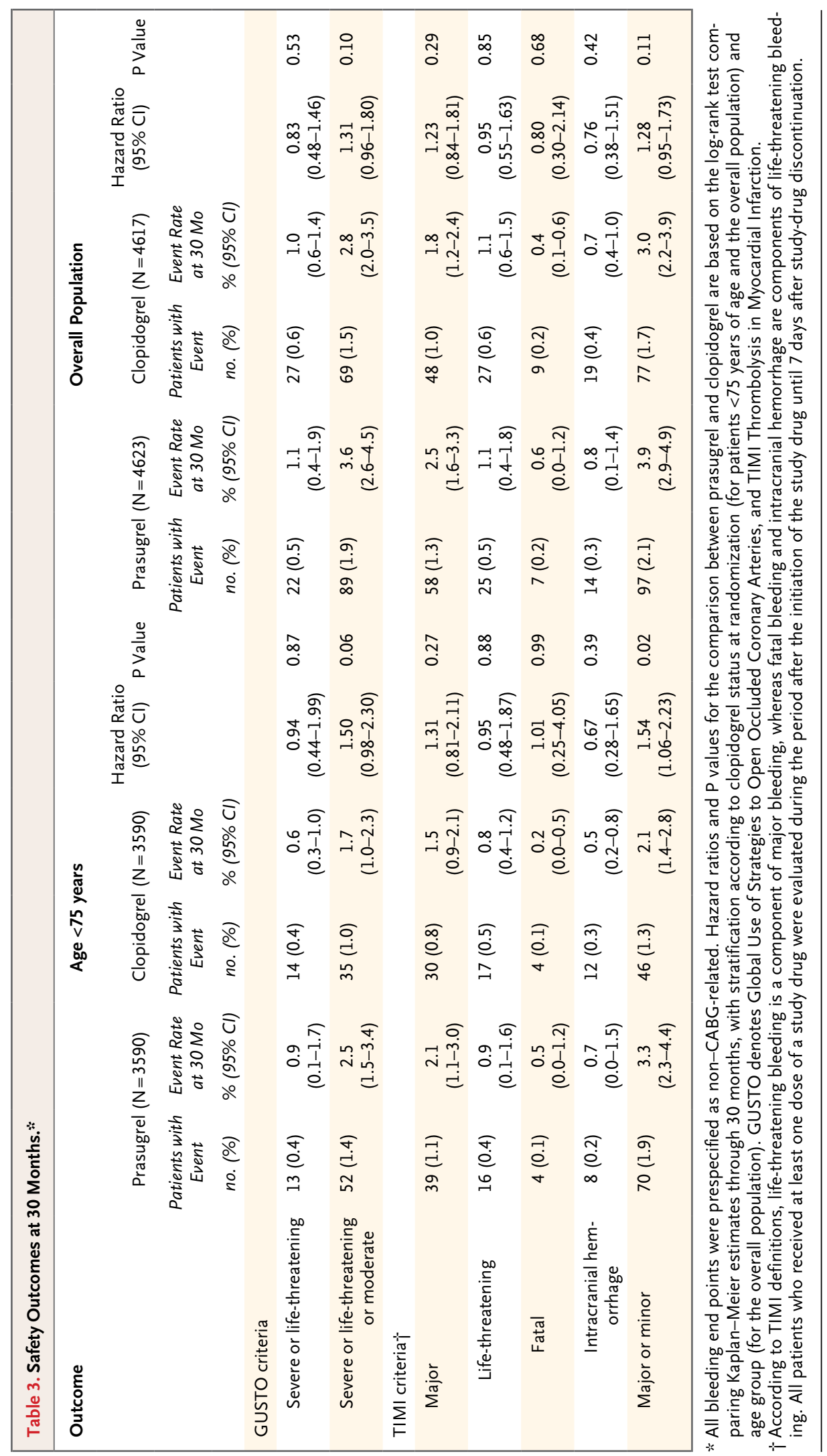


(according to TIMI criteria) occurred with similar frequency among patients under the age of 75 years in the two study groups (Table 3). The KaplanMeier curves for TIMI major bleeding appeared to diverge slightly between study groups after 30 days but remained parallel thereafter (Fig. 1A). The only subgroup in which there was a significant treatment interaction for TIMI major bleeding was patients receiving a reduced dose of aspirin (Fig. S3 in the Supplementary Appendix). Curves for bleeding events in the overall population remained parallel throughout the study (Fig. S2 in the Supplementary Appendix). TIMI life-threatening, fatal, or intracranial bleeding occurred infrequently, and rates were balanced in the two study groups, both in patients under the age of 75 years and in the overall population. Among the younger patients, rates of non-CABG-related severe or lifethreatening or moderate bleeding (GUSTO criteria) and major or minor bleeding (TIMI criteria) were higher in the prasugrel group.

The frequency of new, nonbenign neoplasms in the overall treated population did not differ significantly between the prasugrel group and the clopidogrel group ( $1.9 \%$ vs. $1.8 \%, \mathrm{P}=0.79$ ); similar findings were observed among treated patients with no history of cancer or a history of previous cancer that had been cured before randomization (1.8\% vs. $1.7 \%, \mathrm{P}=0.79$ ).

The incidence of common $(>1 \%)$ nonhemorrhagic serious adverse events was balanced between the two study groups among patients under the age of 75 years, and the only significant difference observed was a higher rate of heart failure in the clopidogrel group (Table S3 in the Supplementary Appendix).

\section{DISCUSSION}

In this large, randomized trial of prolonged treatment with prasugrel, as compared with clopidogrel, in patients with unstable angina or myocardial infarction without ST-segment elevation who did not undergo revascularization, we did not find a reduction in the rate of major cardiovascular events in the prasugrel group. The more intense platelet inhibition with prasugrel was confirmed by the observation of higher rates of minor or moderate bleeding among patients receiving prasugrel, although there was no significant increase in the rate of severe, major, or life-threatening bleeding despite treatment for up to 30 months.
An unexpected time-dependent divergence of treatment effect was observed after 12 months of therapy among patients under the age of 75 years. When evaluated before and after 12 months, the interaction of the treatment effect of prasugrel for the time to the first event was weak, but the late separation of the event curves was consistent for both primary and component end points, an observation that was also apparent in the analysis of multiple recurrent ischemic events. The reasons for this finding remain uncertain, since there have been few studies focusing on high-risk patients who did not undergo revascularization. Such patients would be expected to have a more linear and sustained risk of ischemic events that is not provoked by the use of revascularization procedures during the index hospitalization. A prospective natural-history study of coronary atherosclerosis after an acute coronary event supported this concept, since it showed a near-linear event rate over a 3 -year period, with almost $50 \%$ of later cardiovascular events occurring in nontarget vessels. ${ }^{13}$ Furthermore, platelet reactivity may be amplified after revascularization procedures, so an early response to intensified platelet inhibition may not be apparent in patients receiving medical therapy, as has been shown in patients undergoing invasive procedures. ${ }^{8,9,14}$ Consequently, it is possible that a median follow-up period of 17 months was not long enough to explore the divergence of ischemic events in patients receiving medical therapy alone.

The significant treatment effect of prasugrel on multiple recurrent ischemic events in this study is consistent with findings from the Trial to Assess Improvement in Therapeutic Outcomes by Optimizing Platelet Inhibition with Prasugrel (TRITON), in which the relative risk of recurrent ischemic events was reduced by $30 \%$ in the prasugrel group among patients treated with PCI. ${ }^{15}$ The majority of the effect of prasugrel on recurrent ischemic events occurred later in both trials, but the degree of late separation appeared to be more pronounced in our study. Although this observation is exploratory, it raises the question of whether investigation of the multiplicity of ischemic events is warranted in future secondaryprevention trials, rather than solely analyzing the time to the first event, as has been traditional in studies involving patients who have had an acute coronary event.

This trial had a long follow-up (up to 2.5 years) among patients receiving prasugrel after an acute 


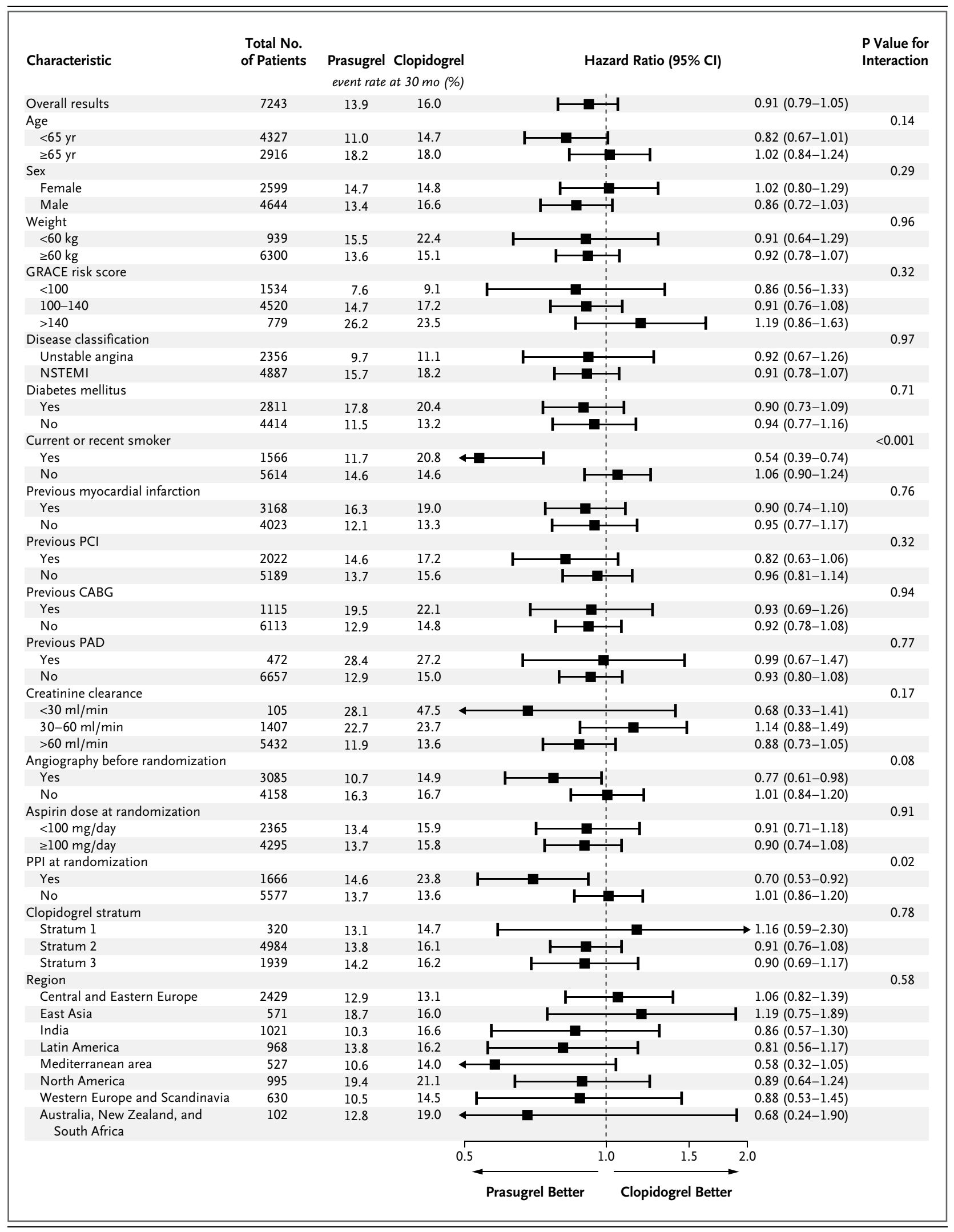


Figure 2 (facing page). Hazard Ratios and Rates of the Primary Efficacy End Point in Prespecified Subgroups of Patients under the Age of 75 Years.

Shown are Kaplan-Meier estimates of the rate of the primary end point through 30 months according to study-group assignment. $P$ values for interaction are shown next to the respective event rates for each designated subgroup. CABG denotes coronary-artery bypass grafting, GRACE Global Registry of Acute Coronary Events, NSTEMI non-ST-segment elevation myocardial infarction, PAD peripheral arterial disease, $\mathrm{PCl}$ percutaneous coronary intervention, and PPI proton-pump inhibitor. Current or recent smokers were defined as patients who smoked cigarettes within 30 days before randomization. Clopidogrel strata are defined as follows: stratum 1, no clopidogrel administered before randomization, with randomization occurring within 72 hours after the first medical contact and if assigned to the clopidogrel group, starting with a loading dose of $300 \mathrm{mg}$ of clopidogrel; stratum 2, loading dose of clopidogrel ( 300 to $600 \mathrm{mg}$ ) administered for the index event, followed by a daily clopidogrel maintenance dose $(75 \mathrm{mg})$ until the day of randomization; and stratum 3, a daily maintenance dose of clopidogrel $(75 \mathrm{mg})$, starting at least 5 days before presentation for the index event and continuing until the day of randomization.

coronary event in a population at high risk for major bleeding events. In TRITON, more serious or life-threatening bleeding events were seen in the prasugrel group, ${ }^{15}$ a finding that was not observed in our study. The risk of major bleeding was low and was similar in the two study groups, but the prasugrel dose was adjusted for patients who were 75 years of age or older and for those weighing less than $60 \mathrm{~kg}$ in order to mitigate such risks. Nonetheless, a biologic effect throughout the period of prasugrel exposure is supported by the increased frequency of minor or moderate bleeding that was observed in this group. Finally, the prospective, systematic surveillance and rigorous adjudication of new, nonbenign neoplasms in our study showed no increase in the risk of neoplasm development with sustained exposure to prasugrel for up to 2.5 years.

Prasugrel was not shown to be superior to clopidogrel for reducing the primary end point during 2.5 years of follow-up after a coronary event in patients receiving medical therapy without planned revascularization, even though signs of intensified platelet inhibition were observed in the prasugrel group. The optimal treatment duration and intensity of $\mathrm{P}_{2} \mathrm{Y}_{12}$ inhibition after a coronary event for patients who do not undergo revascularization remain uncertain. However, our findings highlight the need for further study of differences in the response to intensified platelet inhibition for patients receiving medical therapy without revascularization, as compared with those undergoing revascularization, for treatment of an index cardiac event.

Supported by Eli Lilly and Daiichi Sankyo.

Dr. Roe reports receiving grant support from Eli Lilly, BristolMyers Squibb, Hoffmann-La Roche, Novartis, Schering-Plough, and KAI Pharmaceuticals, consulting fees from Eli Lilly, Daiichi Sankyo, Bristol-Myers Squibb, GlaxoSmithKline, Merck, Janssen Pharmaceuticals, KAI Pharmaceuticals, Sanofi-Aventis, Helsinn Pharmaceuticals, Regeneron, Novartis, AstraZeneca, and Orexigen, and lecture fees from AstraZeneca and Janssen Pharmaceuticals; Dr. Armstrong, receiving consulting fees from Eli Lilly, HoffmannLa Roche, Merck, Axio Research, and Orexigen, grant support from Boehringer Ingelheim, Hoffmann-La Roche, Sanofi-Aventis, Scios, Ortho Biotech, Johnson \& Johnson, Janssen Pharmaceuticals, GlaxoSmithKline, Amylin Pharmaceuticals, and Merck, and payment for developing educational presentations from AstraZeneca and Eli Lilly; Dr. Fox, receiving grant support from Eli Lilly, Bayer, Johnson \& Johnson, and AstraZeneca, travel expenses from Eli Lilly, consulting fees from Eli Lilly, Bayer, Johnson \& Johnson, AstraZeneca, Sanofi-Aventis, and Boehringer Ingelheim, and lecture fees from Bayer, Johnson \& Johnson, AstraZeneca, and Sanofi-Aventis; Dr. White, receiving grant support from Sanofi-Aventis, Eli Lilly, the Medicines Company, Pfizer, Roche, Johnson \& Johnson, Schering-Plough, Merck, AstraZeneca, GlaxoSmithKline, Daiichi Sankyo, and Bristol-Myers Squibb and serving as a board member for Merck and Regado Biosciences; Dr. Dorairaj Prabhakaran, receiving consulting fees from Eli Lilly; Dr. Goodman, receiving grant support from Eli Lilly, Sanofi-Aventis, Bristol-Myers Squibb, and AstraZeneca, consulting fees from Eli Lilly, Sanofi-Aventis, Bristol-Myers Squibb, and AstraZeneca, and payment for developing presentations and lecture fees from Eli Lilly, Sanofi-Aventis, Bristol-Myers Squibb, and AstraZeneca; Dr. Cornel, serving as a board member for Eli Lilly, Daiichi Sankyo, and AstraZeneca and receiving consulting fees from Merck; Dr. Bhatt, serving as a board member for Medscape Cardiology, Boston VA Research Institute, and the Society of Chest Pain Centers, receiving grant support from Amarin, AstraZeneca, Bristol-Myers Squibb, Eisai, Ethicon, Medtronic, Sanofi-Aventis, and the Medicines Company, receiving payment for developing educational presentations from WebMD, and serving as an editor for the American College of Cardiology and chief medical editor for Slack Publications; Dr. Clemmensen, receiving grant support from Eli Lilly and Daiichi Sankyo, consulting fees from Eli Lilly, Daiichi Sankyo, AstraZeneca, Bayer, Pfizer, and Boehringer Ingelheim, and lecture fees from BristolMyers Squibb, having an equity interest in True Green Pharma and Tadpole, and receiving travel expenses from Pfizer, BristolMyers Squibb, AstraZeneca, Bayer, the Medicines Company, and Eli Lilly; Dr. Martinez, receiving consulting and travel fees from Eli Lilly and Daiichi Sankyo; Dr. Ardissino, receiving consulting and lecture fees from Daiichi Sankyo and Eli Lilly; Dr. Nicolau, receiving grant support from Eli Lilly, AstraZeneca, Johnson \& Johnson, Bristol-Myers Squibb, Astellas, Sanofi-Aventis, and Daiichi Sankyo, serving as a board member for AstraZeneca, Bayer, and Sanofi-Aventis, and receiving lecture fees from AstraZeneca, Bayer, and Sanofi-Aventis; Dr. Boden, receiving travel expenses from Eli Lilly and lecture fees from Abbott Laboratories and Gilead Sciences; Dr. Gurbel, receiving consulting fees from Daiichi Sankyo, Eli Lilly, Pozen, Novartis, Bayer, AstraZeneca, Accumetrics, Nanosphere, Sanofi-Aventis, Boehringer Ingelheim, Merck, Iverson Genetics, Medtronic, CSL, and Haemonetics, grant support from Daiichi Sankyo, Eli Lilly, Pozen, CSL, AstraZeneca, Sanofi-Aventis, Haemoscope, and Medtronic, lecture 
fees from Eli Lilly, Daiichi Sankyo, Nanosphere, Sanofi-Aventis, Merck, and Iverson Genetics, and fees for developing educational presentations from Schering-Plough, Discovery Channel, Pri-Med, Merck, Medtronic, and Pfizer; Dr. Dalby, serving as a board member for Sanofi-Aventis, Eli Lilly, Novartis, Boehringer Ingelheim, and Aspen, and receiving travel expenses from AstraZeneca, Merck, Sanofi-Aventis, ICON, Astellas, Daiichi Sankyo, and Novartis; Dr. McGuire, receiving grant support, travel expenses, and fees for review activities from Eli Lilly and Daiichi Sankyo and receiving consulting fees from Tethys Bioscience, Daiichi Sankyo, Genentech, Pfizer, Sanofi-Aventis, Regeneron, and Johnson \& Johnson; Dr. Parkhomenko, receiving consulting fees from AstraZeneca, Merck, Bayer, Takeda, and GlaxoSmithKline, grant support from BHFZ, Servier, Sanofi-Aventis, Astellas, Takeda, Boehringer Ingelheim, and GlaxoSmithKline, lecture fees from Servier, Orion, GlaxoSmithKline, and Pfizer, and payment for development of educational presentations from Servier and Pfizer; Dr. Gottlieb, receiving travel expenses from Eli Lilly and Daiichi Sankyo and consulting fees from Eli Lilly; Dr. Hamm, consulting fees from Medtronic, Hoffmann-La Roche, Brahms, AstraZeneca, and Boehringer Ingelheim, serving as a board member for Medtronic, Hoffmann-La Roche, Brahms, AstraZeneca, and Boehringer Ingelheim, and receiving lecture fees from AstraZeneca, Abbott, Bayer, GlaxoSmithKline, Boehringer Ingelheim, Eli Lilly, Daiichi Sankyo, Abbott, HoffmannLa Roche, Boston Scientific, Sanofi-Aventis, and the Medicines Company; Dr. Pavlides, receiving grant support from Eli Lilly; Dr. Oto, receiving grant support, consulting fees, and travel expenses from Eli Lilly; Dr. Merkely, receiving consulting fees and travel expenses from Eli Lilly and Daiichi Sankyo; Dr. Gašparović, receiving grant support from the Croatian Ministry of Science and Fresenius, consulting fees from Quintiles Eastern
Holdings, travel expenses from Eli Lilly and Merck, grant support from Quintiles Eastern Holdings and Pfizer, and lecture fees from Bayer; Dr. Corbalan, receiving grant support, consulting fees, and travel expenses from Eli Lilly and Daiichi Sankyo; Dr. Cinteză, receiving grant support and consulting fees from Eli Lilly and AstraZeneca and travel expenses and review activity fees from Eli Lilly; Mr. McLendon, receiving travel expenses from Eli Lilly; Drs. Winters and Brown, being employees of and having an equity interest in Eli Lilly; Dr. Lokhnygina, receiving grant support, travel expenses, and fees for review activities from Eli Lilly and Daiichi Sankyo, consulting fees from Johnson \& Johnson, and grant support from Johnson \& Johnson, Merck, Regado Biosciences, Schering-Plough, and Bristol-Myers Squibb; Dr. Aylward, receiving grant support from Eli Lilly, Merck, Bayer, and Johnson \& Johnson, travel expenses from Eli Lilly, AstraZeneca, and Boehringer Ingelheim, and consulting and lecture fees from Eli Lilly, AstraZeneca, Boehringer Ingelheim, Pfizer, Sanofi-Aventis, and Bayer; Dr. Hochman, receiving consulting fees from Eli Lilly and GlaxoSmithKline; and Dr. Ohman, receiving grant support and travel expenses from Daiichi Sankyo and Eli Lilly, consulting fees from AstraZeneca, Boehringer Ingelheim, Bristol-Myers Squibb, Gilead Sciences, Janssen Pharmaceuticals, Liposcience, Merck, Pozen, Hoffmann-La Roche, Sanofi-Aventis, the Medicines Company, and Web MD, grant support from Gilead Sciences, and lecture fees from Gilead Sciences, Boehringer Ingelheim, and the Medicines Company.

Disclosure forms provided by the authors are available with the full text of this article at NEJM.org.

We thank Karen Pieper, M.S., for managing the statistical analysis team; Jonathan McCall, M.S., for providing editorial assistance; and Kerry Stenke for providing graphics assistance.

\section{APPENDIX}

The authors' affiliations are as follows: Duke Clinical Research Institute (M.T.R., R.C.M., Y.L., E.M.O.) and the Division of Cardiology, Department of Medicine (M.T.R., E.M.O), Duke University Medical Center, Durham, NC; the Division of Cardiology, University of Alberta, Edmonton (P.W.A.), and the Division of Cardiology, Department of Medicine, St. Michael's Hospital, University of Toronto, Toronto (S.G.G.) — both in Canada; Centre for Cardiovascular Science, University of Edinburgh, Edinburgh (K.A.A.F.); Auckland City Hospital, Green Lane Cardiovascular Service, Auckland, New Zealand (H.D.W.); Center for Chronic Disease Control, New Delhi, India (D.P.); the Department of Cardiology, Medical Center Alkmaar, Alkmaar, the Netherlands (J.H.C.); VA Boston Healthcare System, Brigham and Women's Hospital, and Harvard Medical School, Boston (D.L.B.); Rigshospitalet, University of Copenhagen, Copenhagen (P.C.); the Department of Cardiology, Cordoba National University, Cordoba, Argentina (F.M.); the Division of Cardiology, Azienda Ospedaliero-Universitaria di Parma, Parma, Italy (D.A.); Heart Institute (InCor), University of São Paulo Medical School, São Paulo (J.C.N.); the Department of Medicine, Stratton VA Medical Center/Albany Medical College, Albany (W.E.B.), and Cardiovascular Clinical Research Center, Leon H. Charney Division of Cardiology, New York University School of Medicine and NYU Langone Medical Center (J.S.H.) - both in New York; Sinai Center for Thrombosis Research, Sinai Hospital of Baltimore, Baltimore (P.A.G.); the Department of Coronary Artery Disease, Institute of Cardiology, Warsaw, Poland (W.R.); Milpark Hospital, Johannesburg (A.J.D.); University of Texas-Southwestern Medical Center, Dallas (D.K.M.); Cardiology Department, Hospital Central "Dr. Morones Prieto," San Luis Potosi, Mexico (J.L.L.-P.); Emergency Cardiology, Institute of Cardiology, Kiev, Ukraine (A.P.); the Department of Cardiology, Bikur Cholim Hospital, Jerusalem (S.G.); the Department of Medicine, Medical Center Manila, Manila, Philippines (G.O.T.); Kerckhoff Heart Center, Bad Nauheim, Germany (C.H.); Cardiology Division, Onassis Cardiac Surgery Center, Kallithea, Greece (G.P.); Cardiology Department, Queen Giovanna University Hospital, Sofia, Bulgaria (A.R.G.); the Department of Cardiology, Hacettepe University Faculty of Medicine, Ankara, Turkey (A.O.); the Division of Cardiology, National Taiwan University College of Medicine, Taipei, Taiwan (C.-D.T.); Heart Center, Semmelweis University, Budapest, Hungary (B.M.); the Department of Intensive Care Medicine, Clinical Hospital Center Zagreb, Zagreb, Croatia (V.G.); Cardiovascular Division, Faculty of Medicine, Pontificia Universidad Católica de Chile, Santiago, Chile (R.C.); the Division of Cardiology, Emergency University Hospital of Bucharest, Bucharest, Romania (M.C.); Eli Lilly, Indianapolis (K.J.W., E.B.B.); South Australian Health and Medical Research Institute, Flinders University Medical Centre, Adelaide, SA, Australia (P.E.A.); and Third Department of Medicine, Cardiology and Emergency Medicine, Wilhelminenhospital, Vienna (K.H.).

\section{REFERENCES}

1. Wright RS, Anderson JL, Adams CD, et al. 2011 ACCF/AHA focused update of the guidelines for the management of patients with unstable angina/non-ST-elevation myocardial infarction (updating the 2007 guideline): a report of the American College of Cardiology Foundation/American Heart Association Task Force on Prac- tice Guidelines developed in collaboration with the American College of Emergency Physicians, Society for Cardiovascular Angiography and Intervention, and Society of Thoracic Surgeons. J Am Coll Cardiol 2011;57:1920-59. [Erratum, J Am Coll Cardiol 2011;57:1960.]

2. Hamm CW, Bassand JP, Agewall S, et al.
ESC guidelines for the management of acute coronary syndromes in patients presenting without persistent ST-segment elevation. Eur Heart J 2011;32:2999-3054. 3. Chan MY, Mahaffey KW, Sun LJ, et al. Prevalence, predictors, and impact of conservative medical management for patients with non-ST-segment elevation acute cor- 
onary syndromes who have angiographically documented significant coronary disease. JACC Cardiovasc Interv 2008;1: 369-78. [Erratum, JACC Cardiovasc Interv 2008;1:600-1.]

4. Goto K, Lansky AJ, Fahy M, et al. Predictors of outcomes in medically treated patients with acute coronary syndromes after angiographic triage: an Acute Catheterization And Urgent Intervention Triage Strategy (ACUITY) substudy. Circulation 2010;121:853-62.

5. Roe MT, White JA, Kaul P, et al. Regional patterns of use of a medical management strategy for patients with non-ST-segment elevation acute coronary syndromes: insights from the EARLY ACS Trial. Circ Cardiovasc Qual Outcomes 2012;5:205-13.

6. Roe MT, Messenger JC, Weintraub WS, et al. Treatments, trends, and outcomes of acute myocardial infarction and percutaneous coronary intervention. J Am Coll Cardiol 2010;56:254-63.
7. Tricoci P, Huang $Z$, Held $C$, et al. Thrombin-receptor antagonist vorapaxar in acute coronary syndromes. $\mathrm{N}$ Engl $\mathrm{J}$ Med 2012;366:20-33.

8. Wallentin L, Becker RC, Budaj A, et al. Ticagrelor versus clopidogrel in patients with acute coronary syndromes. $\mathrm{N}$ Engl J Med 2009;361:1045-57.

9. Wiviott SD, Braunwald E, McCabe $\mathrm{CH}$, et al. Prasugrel versus clopidogrel in patients with acute coronary syndromes. N Engl J Med 2007;357:2001-15.

10. Chin CT, Roe MT, Fox KA, et al. Study design and rationale of a comparison of prasugrel and clopidogrel in medically managed patients with unstable angina/ non-ST-segment elevation myocardial infarction: the TaRgeted platelet Inhibition to cLarify the Optimal strategy to medically manage Acute Coronary Syndromes (TRILOGY ACS) trial. Am Heart J 2010; 160(1):16.e1-22.e1.

11. Riesmeyer JS, Salazar DE, Weerakkody GJ, et al. Relationship between expo- sure to prasugrel active metabolite and clinical outcomes in the TRITON-TIMI 38 substudy. J Clin Pharmacol 2012;52:789-97. 12. Andersen PK, Gill RD. Cox's regression model for counting processes: a large sample study. Ann Stat 1982;10:1100-20.

13. Stone GW, Maehara A, Lanksy AJ, et al. A prospective natural-history study of coronary atherosclerosis. N Engl J Med 2011;364:226-35. [Erratum, N Engl J Med 2011;365:2040.]

14. Gurbel PA, Bliden KP, Hiatt BL, O'Connor CM. Clopidogrel for coronary stenting: response variability, drug resistance, and the effect of pretreatment platelet reactivity. Circulation 2003;107: 2908-13.

15. Murphy SA, Antman EM, Wiviott SD et al. Reduction in recurrent cardiovascular events with prasugrel compared with clopidogrel in patients with acute coronary syndromes from the TRITON-TIMI 38 trial. Eur Heart J 2008;29:2473-9.

Copyright () 2012 Massachusetts Medical Society. 